\title{
Design of surface forces apparatus for tribology studies combined with nonlinear optical spectroscopy
}

\author{
P. Frantz, ${ }^{\text {a) }}$ F. Wolf, ${ }^{\text {b) }}$ X.-d. Xiao, ${ }^{\text {c) }}$ Y. Chen, S. Bosch, ${ }^{\text {d) }}$ and M. Salmeron \\ Materials Sciences Division, Lawrence Berkeley National Laboratory, University of California, Berkeley, \\ California 94720
}

(Received 9 December 1996; accepted for publication 27 March 1997)

\begin{abstract}
We describe the design, calibration, and performance of surface forces apparatus with the capability of illumination of the contact interface for spectroscopic investigation using optical techniques. The apparatus can be placed in the path of a Nd-YAG laser for studies of the linear response or the second harmonic and sum-frequency generation from a material confined between the two surfaces. In addition to the standard fringes of equal chromatic order technique, which we have digitized for accurate and fast analysis, the distance of separation can be measured with a fiber-optic interferometer during spectroscopic measurements ( $2 \AA$ resolution and $10 \mathrm{~ms}$ response time). The sample approach is accomplished through application of a motor drive, piezoelectric actuator, or electromagnetic lever deflection for variable degrees of range, sensitivity, and response time. To demonstrate the operation of the instrument, the stepwise expulsion of discrete layers of octamethylcyclotetrasiloxane from the contact is shown. Lateral forces may also be studied by using piezoelectric bimorphs to induce and direct the motion of one surface. (C) 1997 American Institute of Physics. [S0034-6748(97)05906-6]
\end{abstract}

\section{INTRODUCTION}

The surface forces apparatus (SFA) has demonstrated extensive utility as a tool to study static and dynamic forces between molecularly smooth, macroscopic surfaces. ${ }^{1-3}$ With this instrument, two freshly cleaved mica surfaces oppose each other in a crossed cylinder geometry to produce a circular contact that is nearly ideal, and mechanical and chemical properties may be manipulated and characterized with great precision. Recent developments of the apparatus have allowed it to contribute to the blossoming field of molecular tribology - the study of tribology at the atomic and molecular scale. ${ }^{4}$ These modifications permit sensitive measurements of shear forces as one surface is moved laterally with respect to the other. By observing the behavior of a material interposed between mica sheets during shear, it has been shown that creation and subsequent changes in the molecular organization may be responsible for many fundamental tribological phenomena such as the finite yield stress of a confined liquid. 1,4

A shortcoming of all previous experiments is that molecular organization must be inferred from measurements of the tribological properties that they influence. The absence of direct observations of molecular arrangements has been particularly notorious. Progress has been slow because a formidable obstacle arises when attempting to couple the conventional SFA with any form of spectroscopy. For typical SFA measurements, it is necessary to deposit a semireflective

\footnotetext{
a) Present address: Surface Science Department, The Aerospace Corporation, 2350 E. El Segundo Boulevard, El Segundo, California 90245.

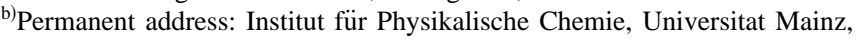
D-55099 Mainz, Germany.

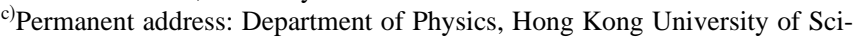
ence and Technology, Clear Water Bay, Kowloon, Hong Kong.

${ }^{d)}$ Permanent address: Department of Fisica Aplicada i Electrònica, Universitat de Barcelona, 08028 Barcelona Spain.
}

layer of silver on the backside of the mica sheets. ${ }^{3}$ These reflective surfaces create the interference cavity of a FabryPerot interferometer to provide a measure of the distance of separation and the forces applied to the interface. Regrettably, these reflective surfaces do not allow sufficient excitation source intensity to illuminate the buried interface for many forms of optical spectroscopy. In fact, it has been predicted that, due to high absorbance in the infrared, the silver layer will ablate when exposed to laser intensities necessary for measurements of sum-frequency (SFG) or second harmonic generation (SHG). ${ }^{5}$

Recently, spectroscopic probes of the orientation and composition of confined molecules have begun to emerge. Israelachvili and co-workers ${ }^{6}$ have developed an instrument capable of $\mathrm{x}$-ray investigation of the buried interface. In this scheme, $\mathrm{x}$ rays penetrate the silver layers and scatter from the ordered strata of liquid crystals placed between the surfaces. Under shear, the liquid was observed to change orientation. This approach is well suited to the study of liquid crystal molecules which form ordered arrangements when the confining walls are more distant that several molecular diameters. However, since experimental limitations precluded collection of meaningful results when the surfaces were separated by $<0.4 \mu \mathrm{m}$, this technique could not be used to study ultrathin liquid films. Encouraging results have also been obtained by Helm and co-workers, ${ }^{7,8}$ who have measured optical properties of a thin dye layer adsorbed to the mica surfaces of an SFA. They show that the intensity and resonance wavelength transmission peaks from a FabryPerot interferometer contain a wealth of information about the concentration and interactions of confined dye molecules. This technique is limited only by the selection of molecules for study (large dye molecules) and the poor spatial resolution that results from using thick mica (to minimize the spacing of transmission peaks). ${ }^{7}$

It has recently been demonstrated that SHG and SFG are 
ideal tools to study buried interfaces. ${ }^{9,10}$ These techniques can be readily adopted to study molecular films under static pressure to provide molecular orientation or conformational information. In our laboratory, a primitive SFA, consisting of a quartz sphere and optical flat, has been used to study orientation, conformation, and molecular vibrations of a variety of Langmuir-Blodgett (L-B) and SAM films under pressure. ${ }^{10}$ Under a pressure of $50 \mathrm{MPa}$, the SFG and SHG signals were found to decrease by a factor of 100-1000 times the original signal. This indicated a vanishing of the second-order monolayer susceptibility due to disorder of the head groups and/or flattening of the molecular axis so that they lie parallel to the surface. With these encouraging results, it is now our goal to combine the nonlinear optics (NLO) laser system with a more sensitive SFA. Actual implementation of the NLO experiments will be presented in a future article.

In this article, we describe an instrument that employs a fiber-optic interferometer (FOI) as an alternative way to monitor the relative positions of the two surfaces. This modification allows for the absence of the silver coating to permit full illumination of the buried interface for spectroscopy, and it provides a continuous monitor of the distance of separation with resolution equal to that of the fringes of equal chromatic order (FECO) method. Measurements may be made quite rapidly, with time resolution limited only by the response time of the electronics (in our case, $\sim 1 \mathrm{~ms}$ ), allowing for the systematic study of hydrodynamic processes. Furthermore, when the spectroscopy is not required, the FOI makes way for the use of more technically relevant surfaces, which are often opaque.

In addition to the FOI, the use of FECO fringes for routine surface forces measurements has reached an advanced degree of sophistication in this apparatus. We describe the data manipulation procedures which allow us to collect interference fringes with a video camera and digitize them for computer analysis. By doing this, the subjectivity of visual fringe location from a broad transmission peak is completely removed.

\section{DESIGN OF THE APPARATUS}

Crossed cylinders of mica meet at the heart of the apparatus (Fig. 1), supported by quartz lenses of $1 \mathrm{~cm}$ diameter and $1 \mathrm{~cm}$ radius of curvature. The bottom lens is supported by the piezoelectric stages shown in Fig. 2. Vertical motion of this surface is achieved through expansion of a piezoelectric tube (Stavely Instruments, Kennewick, WA) giving sensitivity of $<0.1 \mathrm{~nm}$. Transverse motion of the bottom surface is induced and monitored through deformation of piezoelectric bimorphs (APS, Mackeyville, PA), in an arrangement similar to that devised by Peachey and Granick. ${ }^{11}$ For studies that require liquid immersion of the surfaces, the lower surface support may be easily modified to contain the surfaces and fluid in a stainless steel cup, separated from the piezoelectric components.

The top surface is supported by interchangeable leaf springs which provide spring constants from 50 to 5 $\times 10^{5} \mathrm{~N} / \mathrm{m}$. Coarse manipulation of the upper surface is controlled manually with a micrometer, or by a motorized

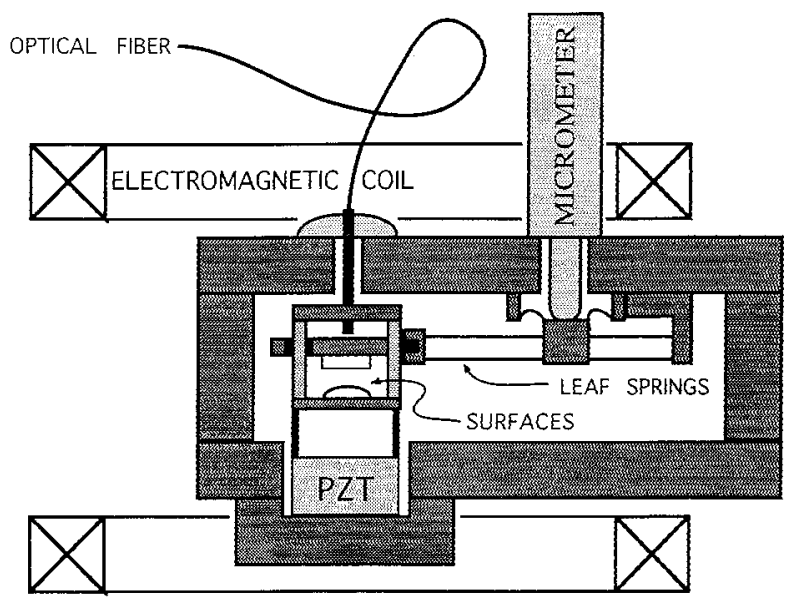

FIG. 1. Schematic of the surface forces apparatus equipped with a fiber optic interferometer and magnetic lever deflection. The black squares mounted beside the upper surface represent magnets which couple to a magnetic field gradient created by the electromagnetic coils for sensitive displacements.

micrometer (Newport Corporation, Irvine, CA), which provides a range of $5 \mathrm{~mm}$ and resolution of $0.5 \mu \mathrm{m}$. The micrometer is isolated from the internal cell of the SFA by a welded stainless steel bellows. Fine adjustments of the upper surface are made with magnetic lever deflection similar to the technique developed by Stewart and Christensen. ${ }^{12}$ In this arrangement, two permanent neodymium-iron-boron magnets (Edmund Scientific Company, Barrington, NJ) are mounted on opposite sides of the upper mica substrate, and they couple to a magnetic field gradient created by an electrical current through two coils of copper wire (300 turns, 39 $\Omega$ ) located outside the box. A current of $100 \mathrm{~mA}$ in the coils creates a force of $1.2 \mathrm{mN}$ on the cantilever. Our current supply has a noise level of $1 \mu \mathrm{A}$, which translates into a smallest applicable force of $0.12 \mathrm{nN}$. This method of position manipulation has the advantage of high reproducibility and lack of hysteresis over microns. In addition, since no motors

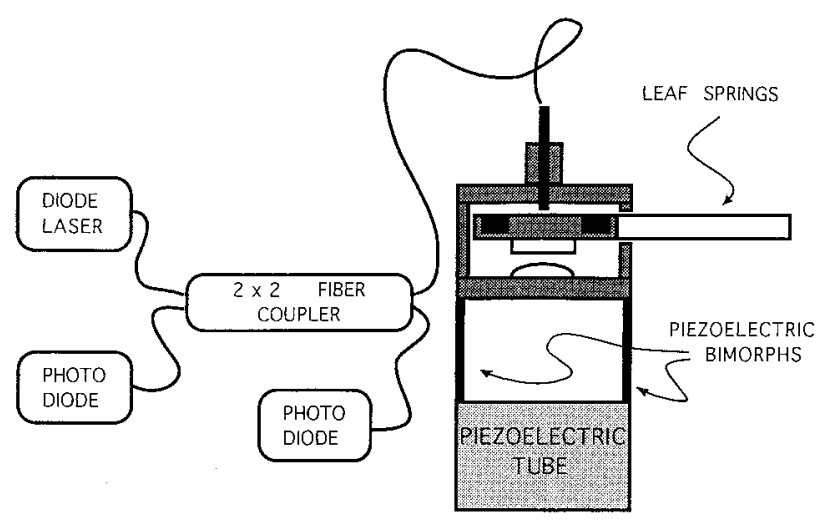

FIG. 2. More detailed schematic of the surface position control devices and the fiber optic interferometer. The bottom surface is sustained by a piezoelectric tube, for fine vertical position control, and piezoelectric bimorphs, for induction and measurement of lateral displacements. An interference cavity exists between the polished end of the fiber and the backside of the upper surface. This fiber is mounted in a piezoelectric tube (not shown) and clamped rigidly to a steel bridge attached to the lower surface support. 
are used, there is no mechanical coupling or movement during approach.

The principles of operation for the FOI are identical to those of many interferometers used in atomic force microscopy (AFM). ${ }^{13}$ Referring to Fig. 2, light from a diode laser is directed through a $2 \times 2$ coupler (Aster Corporation, Hopkinton, MA) where half of the original intensity is directed to each of the outgoing fibers. At the end of one fiber is a photodiode, used for monitoring fluctuations in the intensity from the diode laser. The other fiber is mounted rigidly to the support of the lower surface. This surface support is used as a point of reference for the subsequent motion. Roughly $6 \%$ of the light is internally reflected back into the fiber from the polished end. Of the light that is emitted by the fiber, some amount is reflected from a reflective surface mounted to the back of the upper surface support, and it reenters the fiber from which it was emitted. This secondary reflection interferes with the primary reflection, and the resulting interference intensity is transmitted through the coupler and monitored with a photodiode. The single mode fiber has a diameter of $125 \mu \mathrm{m}$, and the laser diode emits at a wavelength of $780 \mathrm{~nm}$.

The optical fiber is attached rigidly to the bottom surface through a steel support bridge (Fig. 2). Using a micrometerdriven stage outside the apparatus, the polished end of the fiber is placed within $200 \mu \mathrm{m}$ of a reflective surface on the backside of the upper surface. The fiber is then clamped into place before the apparatus is sealed and purged with nitrogen. The fiber is fixed inside a glass capillary tube with UVcured optical cement. This capillary is protected by a steel tube of $1.6 \mathrm{~mm}$ o.d. and the entire assembly is supported by a piezoelectric tube $(12 \mathrm{~mm}$ length $\times 3 \mathrm{~mm}$ o.d.). A fiber splicing kit was used to polish the end of the fiber and glass capillary. The piezoelectric tube allows repositioning of the fiber such that the surface displacement is performed at the maximum slope of the sinusoidal interference fringe.

In an earlier design, the optical fiber was mounted beside the top surface, at a distance of $1 \mathrm{~cm}$ from the contact, to allow direct observation of the contact for simultaneous use of FECO and FOI. However, it was found that mechanical noise levels at that point exceeded $5 \mathrm{~nm}$. Vibrations (rocking motions) about the point of contact could not be avoided. Thus, it was necessary to place the optical fiber within 0.2 $\mathrm{mm}$ of the contact axis to reduce the noise to $0.1 \mathrm{~nm}$, although this obstruction prohibits simultaneous collection of FECO and FOI fringes.

In order to illuminate the contact for spectroscopic investigation, three ports have been installed in the bottom of the apparatus. One large port ( $25 \mathrm{~mm}$ diameter) in the center, along the axis of the substrate support, has been provided for purposes of observation in the alignment process. Two smaller ports (12 $\mathrm{mm}$ diameter), one on each lower corner, allow the laser beam to enter the apparatus through windows at normal incidence, strike the lower surface at $45^{\circ}$, and exit to the detector.

\section{FORCE/DISTANCE MEASUREMENT}

The apparatus acts as a conventional force balance, where the force and relative displacement of the surfaces are

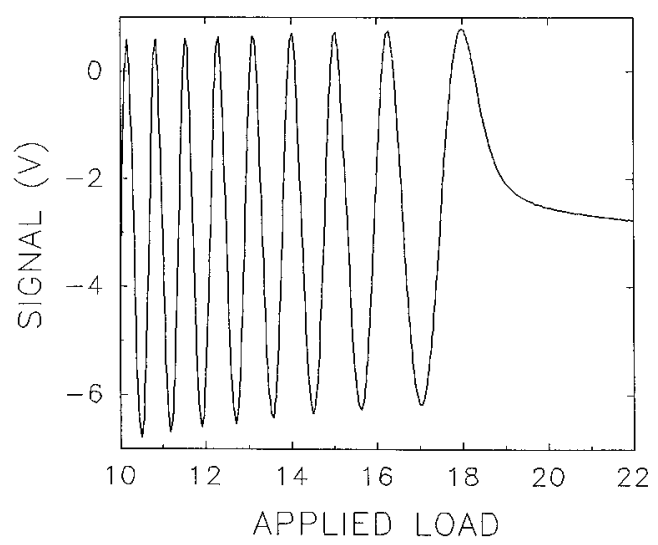

FIG. 3. Sinusoidal fringes from the fiber optic interferometer as undecane is rapidly squeezed from between the surfaces. Signal intensity from the photodiode is plotted against the magnetic force applied to the upper surface. The rate of approach is slowed by hydrodynamic resistance as the surface separation decreases.

related by the spring constant of the leaf springs. As mentioned above, this displacement may be monitored with either the FOI of FECO fringes, depending upon the particular application.

\section{A. Analysis with the fiber-optic interferometer}

As the distance between the surfaces decreases, the distance between the polished end of the fiber and the backside of the top surface increases, and the light which is back reflected through the fiber interferes with the internal reflection from the polished end. The result is a series of sinusoidal fringes. The wavelength of these fringes is equal to one-half of the wavelength of light emitted by the diode laser $(\lambda / 2=390 \mathrm{~nm})$ and the amplitude, which can provide a maximum output of $\sim 10 \mathrm{~V}$ from the photodiode, decreases as the separation increases. This signal is monitored with a voltmeter and recorded digitally in real time on a personal computer.

Figure 3 shows interference fringes during rapid approach of the surfaces with viscous fluid, undecane, interposed between the mica sheets. Here, the photodiode output is plotted against voltage applied to the coils, which has been converted to applied load. The surfaces were initially separated by $3.5 \mu \mathrm{m}$ at the left of the figure, and roughly nine fringes passed as the surfaces were brought into contact. At first, these fringes passed quickly as undecane was expelled from between the surfaces, but the process soon slowed due to the enhanced significance of repulsive hydrodynamic forces when the liquid became severely confined. In an experiment such as this, with a total signal amplitude of $7 \mathrm{~V}$ (per $195 \mathrm{~nm}$ traveled) and a meter sensitivity of better than 1 $\mathrm{mV}$, we have a spatial resolution of $<0.3 \AA$. The actual resolution is, however, limited by mechanical vibration about the point of contact. As demonstrated below, spatial resolution of $\sim 2 \AA$ is easily accessible.

During an experiment in which a typical approach curve is collected, the surfaces are first brought to a separation of $20 \mathrm{~nm}$ with a combination of micrometer and magnet adjustments. To maximize the sensitivity, the length of the piezoelectric tube that supports the optical fiber is adjusted so that 
the observed laser intensity is midway between the extreme values. The surface separation remains the same, but the signal is now positioned at the maximum slope of an interference fringe. Since we are interested in measuring approach curves with a range of $20 \mathrm{~nm}$, only a small fraction of a single fringe is necessary. Over this range of motion, the deviation from linearity due to the sinusoidal fringe shape is $1.6 \%$.

The raw data is collected as signal voltage from the photodiode versus force applied to the magnet. The deviation from linearity in this data, neglecting the sinusoidal fringe shape, is due to the normal force supported by the interposed medium and the deformation of the surfaces, which is accounted for below. A simple calibration is used to convert a change in signal intensity to the actual change in separation.

The contribution to detected motion from deformation of the surfaces deserves note. As the liquid confined between the sheets begins to sustain a normal force, due to ordering or hydrodynamic forces, the compliance of the mica/glue substrate will allow deformation. This deformation will cause an apparent change in separation equal to the sum of the deformation and the actual change in distance between the surfaces. This effect, which is responsible for an error of $\sim 10 \%$ over the range of load applied in a typical approach curve $(30 \mu \mathrm{N})$, is easily accounted for by fitting the early deviation from linearity in the signal versus applied load curve with a function describing the change in contact radius with load at a Hertzian contact ${ }^{14}$

$$
a=(R F / K)^{1 / 3} \text {. }
$$

Here, the radius $R$ of the sphere is commonly $10 \mathrm{~mm}, F$ is the force applied to the substrate support by magnetic lever deflection, starting from the point at which the liquid supports a normal force (where the slope of the interference fringe deviates significantly from linearity) and $K$, the elastic modulus, is an adjustable parameter. We assume that the contact radius is proportional to the square root of the depth of the contact deformation. This least squares fitted curve is then subtracted from the raw data. Since there is no dependence on thin mica with this technique as with the FECO method, thick mica $(>10 \mu \mathrm{m})$ is used to minimize differences in the sample modulus and radius of curvature from one sample to the next.

\section{B. Analysis with FECO fringes}

FECO fringes may be collected with the FOI removed. These fringes are generated in the conventional manner, but they are treated and analyzed in a way that speeds data collection and improves accuracy and resolution. The theory and methods behind generation of FECO fringes has been described previously in great detail. ${ }^{3,15}$ In our system, the output of the spectrometer is detected with a sensitive SIT68 video camera (Dage-MTI Inc., Michigan City, IN) and interfaced to a frame-grabber board (Data Translation Quick Capture SP0219) installed in a PC. Typically, for each data point of an approach curve, 20 of these images are collected, digitized and averaged, the background is subtracted, and the resulting image is stored on a PC. To save time and memory storage space, only the region of interest around the leading edge of the fringe is saved.

During collection of an approach curve, a software routine controls the progress of the experiment. As shown in Fig. 4(a), an interference fringe is collected and stored, the current through the coils is increased to provide an incremental change in force applied to the fixed magnets, a specified time is allowed for equilibration, and the next fringe is collected.

After the experiment, the software automatically extracts the sample separation data from the series of fringes. It first reads the averaged digitized image, which is composed of pixels in columns and rows [Fig. 4(b)]. The position of the center of peak intensity is found for each row at a prespecified peak height. The image is then recomposed with a single point in each row marking the peak center [Fig. 4(c)]. This image is used for further analysis, including determination of distances for approach curves or contact area for measurements of adhesion. When constructing approach curves, the leading pixel of each fringe is chosen by fitting the fringe with a parabolic profile. The numerical procedure used is the singular value decomposition, which is used for least squares fitting of functions that have a linear dependence on their parameters. ${ }^{16}$ The method also gives the quality of the fit. Using previously obtained calibration data for the camera pixel columns, the resulting $x$ values are converted into corresponding wavelengths, from which the sample separation and applied force can then be calculated. ${ }^{3}$

\section{RESULTS}

Our primary goal in the development of this apparatus has been to remotely detect molecular scale changes in the separation between the surfaces, while leaving an unobstructed path to the buried interface for laser illumination. To demonstrate the achievement of this goal, the stepwise expulsion of discrete molecular layers of octamethylcyclotetrasiloxane (OMCTS) has been observed using the FOI method. This simple, nonpolar, Newtonian, low molecular weight relative of the silicon oils has been extensively studied in previous surface force measurements. ${ }^{17,18}$ It is roughly spherical with a convenient diameter $(8.5 \mathrm{~nm}) .{ }^{17}$ All liquids were used as received. To begin the experiment, two bare mica surfaces, freshly cleaved in a dry nitrogen chamber, were brought into contact. The zero point photodiode signal at bare mica/mica contact was recorded, the surfaces were carefully separated, and a small drop of OMCTS was injected between them. The surfaces were brought to within 20 $\mathrm{nm}$ separation, and a linearly increasing dc current was applied to the electromagnetic coils. The ramp rate, $0.5 \mathrm{Amp} / \mathrm{s}$, was chosen to provide $\sim 6 \mathrm{~s}$ for collection of the entire approach curve.

Figure 5 shows the result of this approach after correction for substrate deformation. Starting from the left side of this figure, while the surfaces were separated by $>5$ molecular diameters of OMCTS, we observed a rapid change of separation with increasing current applied to the magnet, equal to the slope of the interference fringe when the surfaces were distant. Upon further approach, a small step was 


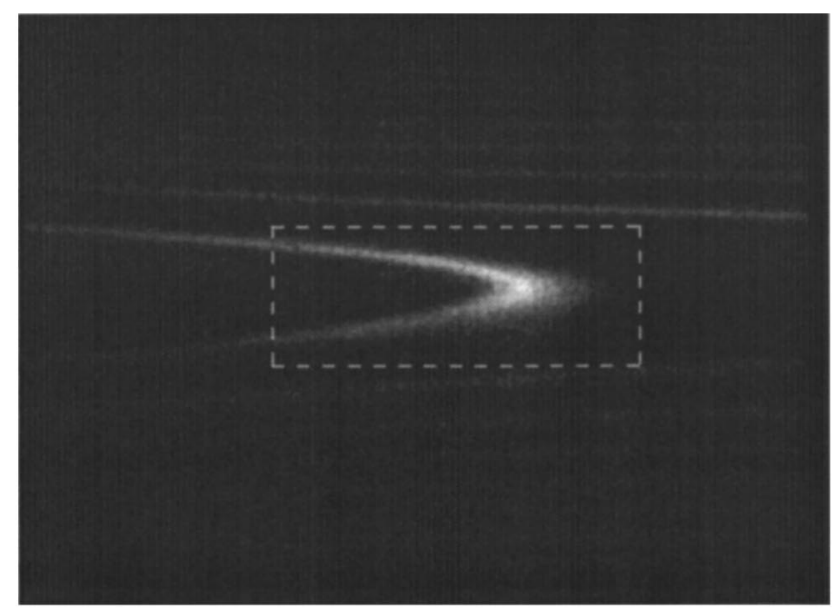

(a)

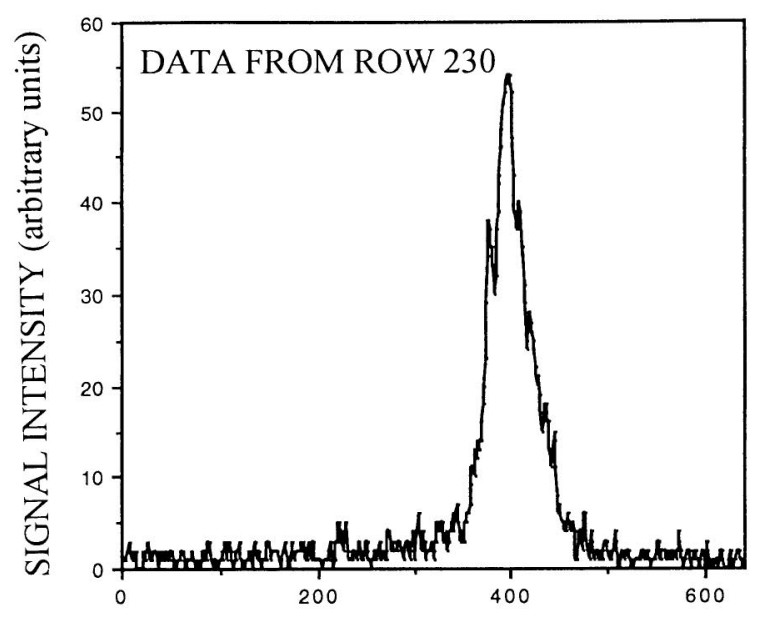

(b)

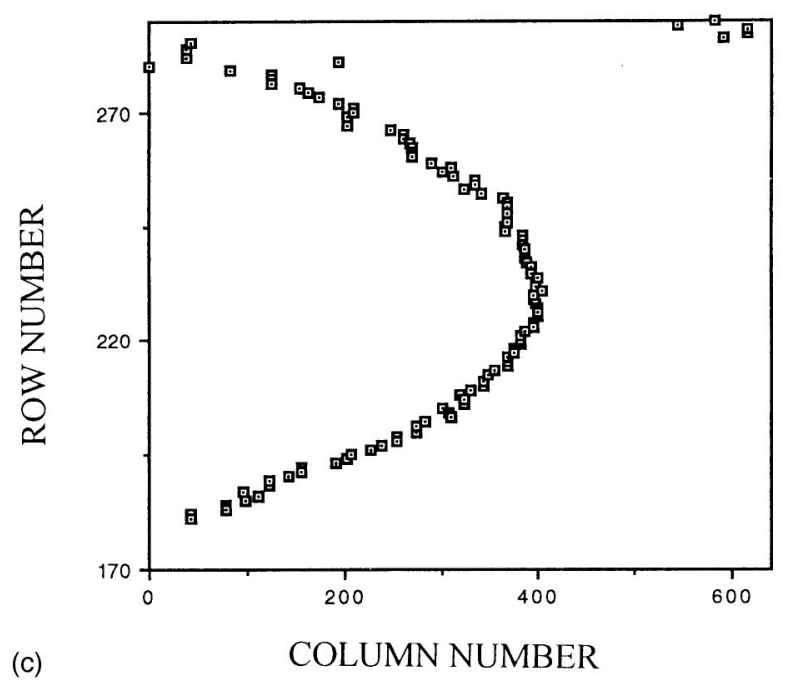

(c)

FIG. 4. (a) Digitized image of a FECO fringe with the surfaces close to contact. The dashed square represents the area saved for further analysis. (b) Pixel intensity vs horizontal position in a line crossing the fringe shown in the box in (a). (c) The center of each peak in (b) is chosen by selecting the midpoint of the peak at a predetermined height. These midpoints are collected for each column and plotted together to give a precise representation of the fringe. This curve is then fitted by a parabolic function, and the apex of the parabola is selected as the leading edge of the fringe. In this matter, subjective visual interpretation of the fringe location is removed.

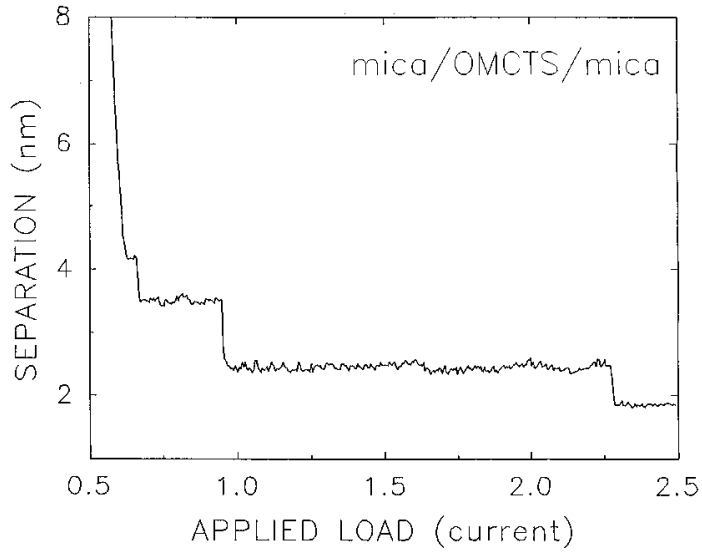

FIG. 5. Change in surface separation with current applied to the electromagnetic coils as OMCTS is expelled in discrete layers from the contact. The average height of each step, $8 \AA$, is approximately equal to the molecular diameter.

observed at a separation of $4.2 \mathrm{~nm}$, roughly 5 molecular diameters, where the fluid first showed evidence of assembly into a solidlike arrangement. As the load is increased, more layers of OMCTS were expelled, until only two layers remain under a load $>20 \mu \mathrm{N}$. These two layers remained at the interface up until a load of $50 \mu \mathrm{N}$, the maximum that was applicable with our device.

We have observed similar steps for other liquids, such as hexadecane and undecane. We have also shown that, under similar environmental conditions, smaller increments of applied load during the approach can yield more steps at greater distances of separation between the surfaces. ${ }^{18}$

These data demonstrate excellent spatial resolution, $\sim 2$ $\AA$, which is limited by mechanical vibrations about the point of contact. The time resolution limited by the response time of our electronics is unprecedented; in this case, each data point is separated by $10 \mathrm{~ms}$. This rapid rate of data collection, which greatly exceeds that of the FECO method, open the door to hydrodynamic studies of liquids as they evacuate confined spaces.

\section{DISCUSSION}

The fiber optic interferometer is a viable means to monitor the distance of separation in a surface forces apparatus. It offers excellent spatial and time resolution, allows for removal of obstructions to spectroscopic investigation of the buried interface, and relaxes the constraints on substrate properties. We have shown that it is possible to quantify the number of discrete layers of molecules between the surfaces, and to manipulate this number with great precision.

Our present efforts are directed towards combining this instrument with a preexisting second-order nonlinear optical spectroscope. Of interest are the orientation, alignment, and relaxation modes of molecules that are subjected to normal and lateral forces, and the influence that these properties have on tribological phenomena. 


\section{ACKNOWLEDGMENTS}

This work was supported by the Director, Office of Energy Research, Basic Energy Sciences, Division of Materials Sciences, of the U.S. Department of Energy under Contract No. DE-AC03-76SF00098. S. B. acknowledges the Gaspar de Portola Catalan Studies Program at the University of California at Berkeley.

${ }^{1}$ J. N. Israelachvili, Handbook of Micro/Nano Tribology (Chemical Rubber, New York, 1995).

${ }^{2}$ J. N. Israelachvili, Chemtracts: Anal. Phys. Chem. 1, 1 (1989).

${ }^{3}$ J. N. Israelachvili, J. Colloid Interface Sci. 44, 259 (1973).

${ }^{4}$ S. Granick, Science 253, 1374 (1991).

${ }^{5} \mathrm{Y}$. R. Shen (private communication).

${ }^{6}$ S. H. J. Idziak et al., Science 264, 1915 (1994).
${ }^{7}$ C. Muller, P. Machtle, and C. A. Helm, J. Phys. Chem. 98, 11119 (1994).

${ }^{8}$ P. Machtle, C. Muller, and C. A. Helm, J. Phys. (France) II 4, 481 (1994).

${ }^{9}$ Q. Du, E. Freysz, and Y. R. Shen, Science 264, 826 (1994).

${ }^{10}$ Q. Du et al., Phys. Rev. B 51, 7456 (1995).

${ }^{11}$ J. Peachey, J. Van Alsten, and S. Granick, Rev. Sci. Instrum. 62, 463 (1991).

${ }^{12}$ A. M. Stewart and H. K. Christenson, Meas. Sci. Technol. 1, 1301 (1990).

${ }^{13}$ D. Rugar, H. J. Mamin, R. Erlandsson, J. E. Stern et al., Rev. Sci. Instrum. 59, 2337 (1988).

${ }^{14} \mathrm{~J}$. N. Israelachvili, Intermolecular and Surface Forces, 2nd ed. (Academic, London, 1992).

${ }^{15}$ S. Tolansky, Multiple-Beam Interferometry of Surfaces and Films (Clarendon, Oxford, 1948).

${ }^{16}$ W. H. Press, Numerical Recipes in $C$ : The Art of Scientific Computing (Cambridge University Press, New York, 1988).

${ }^{17}$ H. K. Christenson, J. Chem. Phys. 78, 6906 (1983).

${ }^{18}$ P. Frantz, N. Agrait, and M. Salmeron, Langmuir 12, 3289 (1996). 